\title{
CORRIGENDUM
}

Journal of General Microbiology (1982), 128, 471-476

\section{Restriction Enzyme Analysis of Mitochondrial DNA of Members of the Genus Aspergillus as an Aid in Taxonomy}

The authors' names should read:

M. KOZŁOWSKI, E. BARTNIK AND P. P. STȨPIEŃ 